\title{
Search for TeV gamma ray emission from the Andromeda galaxy
}

\author{
F. A. Aharonian ${ }^{1}$, A. G. Akhperjanian ${ }^{7}$, M. Beilicke ${ }^{4}$, K. Bernlöhr ${ }^{1}$, H. Bojahr ${ }^{6}$, O. Bolz $^{1}$, H. Börst ${ }^{5}$, T. Coarasa ${ }^{2}$,
} J. L. Contreras ${ }^{3}$, J. Cortina ${ }^{2}$, S. Denninghoff ${ }^{2}$, V. Fonseca ${ }^{3}$, M. Girma ${ }^{1}$, N. Götting ${ }^{4}$, G. Heinzelmann ${ }^{4}$, G. Hermann ${ }^{1}$, A. Heusler ${ }^{1}$, W. Hofmann ${ }^{1}$, D. Horns ${ }^{1}$, I. Jung ${ }^{1}$, R. Kankanyan ${ }^{1,7}$, M. Kestel ${ }^{2}$, J. Kettler ${ }^{1}$, A. Kohnle ${ }^{1}$, A. Konopelko ${ }^{1}$, H. Kornmeyer ${ }^{2}$, D. Kranich ${ }^{2}$, H. Krawczynski ${ }^{1, \%}$, H. Lampeitl ${ }^{1,4}$, M. Lopez ${ }^{3}$, E. Lorenz ${ }^{2}$, F. Lucarelli ${ }^{3}$, O. Mang ${ }^{5}$, H. Meyer ${ }^{6}$, R. Mirzoyan ${ }^{2}$, A. Moralejo ${ }^{3}$, E. Ona ${ }^{3}$, M. Panter ${ }^{1}$, A. Plyasheshnikov ${ }^{1, \S}$, G. Pühlhofer ${ }^{1}$, G. Rauterberg ${ }^{5}$, R. Reyes ${ }^{2}$, W. Rhode ${ }^{6}$, J. Ripken ${ }^{4}$, A. Röhring ${ }^{4}$, G. P. Rowell ${ }^{1}$, V. Sahakian ${ }^{7}$, M. Samorski ${ }^{5}$, M. Schilling ${ }^{5}$, M. Siems ${ }^{5}$, D. Sobzynska ${ }^{2, *}$, W. Stamm ${ }^{5}$, M. Tluczykont ${ }^{4}$, H. J. Völk ${ }^{1}$, C. A. Wiedner ${ }^{1}$, and W. Wittek ${ }^{2}$

1 Max Planck Institut für Kernphysik, Postfach 103980, 69029 Heidelberg, Germany

2 Max Planck Institut für Physik, Föhringer Ring 6, 80805 München, Germany

3 Universidad Complutense, Facultad de Ciencias Físicas, Ciudad Universitaria, 28040 Madrid, Spain

${ }^{4}$ Universität Hamburg, Institut für Experimentalphysik, Luruper Chaussee 149, 22761 Hamburg, Germany

5 Universität Kiel, Institut für Experimentelle und Angewandte Physik, Leibnizstraße 15-19, 24118 Kiel, Germany

${ }^{6}$ Universität Wuppertal, Fachbereich Physik, Gaußstr. 20, 42097 Wuppertal, Germany

7 Yerevan Physics Institute, Alikhanian Br. 2, 375036 Yerevan, Armenia

$\S$ On leave from Altai State University, Dimitrov Street 66, 656099 Barnaul, Russia

\% Now at Yale University, PO Box 208101, New Haven, CT 06520-8101, USA

${ }^{*}$ Home institute: University Lodz, Poland

Received 6 November 2002 / Accepted 23 December 2002

\begin{abstract}
Using the HEGRA system of imaging atmospheric Cherenkov telescopes, the Andromeda galaxy (M 31) was surveyed for $\mathrm{TeV}$ gamma ray emission. Given the large field of view of the HEGRA telescopes, three pointings were sufficient to cover all of M31, including also M 32 and NGC 205. No indications for point sources of TeV gamma rays were found. Upper limits are given at a level of a few percent of the Crab flux. A specific search for monoenergetic gamma-ray lines from annihilation of supersymmetric dark matter particles accumulating near the center of M 31 resulted in flux limits in the $10^{-13} \mathrm{~cm}^{-2} \mathrm{~s}^{-1}$ range, well above the predicted MSSM flux levels except for models with pronounced dark-matter spikes or strongly enhanced annihilation rates.
\end{abstract}

Key words. gamma rays: observations - galaxies: individual: M 31 - dark matter

\section{Introduction}

During the last decade, Imaging Atmospheric Cherekov Telescopes (IACTs) have extended the spectrum of astronomical observations into the $\mathrm{TeV}$ energy regime, and a small number of galactic sources such as plerions and Supernova remnants as well as extragalactic sources - active galactic nuclei (AGN) - have been detected and studied (e.g. Weekes 2001). In particular with stereoscopic systems of Cherenkov telescopes, such as the HEGRA telescope system (Daum et al. 1997; Aharonian et al. 1999; Konopelko et al. 1999), surveys of moderately extended regions of the sky have become possible (Aharonian et al. 2001b, 2002). At this stage, and given the survey capability, it seems natural to search for $\mathrm{TeV}$ gamma ray emission not only from distant AGN, but to turn the

Send offprint requests to: $\mathrm{W}$. Hofmann,

e-mail: wh@daniel.mpi-hd.mpg.de instruments to our nearest neighbor galaxy, M31. While - as will be discussed below - one would not expect conventional sources in M31, equivalent, e.g., to the Crab Nebula, to be visible over the distance, there is the possibility of new classes of sources, which have not been discovered in our own Galaxy because they are not particularly prominent in other wavelength regimes, possibly since they are heavily obscured by interstellar matter. While it is hardly possible to survey with Cherenkov telescopes the whole Galactic sky, it is relatively easy to cover all of M 31 in searching for new TeV sources.

In this work, we report on a TeV survey of M 31 using the HEGRA IACT system. We will first briefly summarize the relevant characteristics of M31 in the remainder of this section, and then discuss the data analysis and the results.

Excellent summaries of the characteristics of M31 can be found in (Hodge 1992) and in (Berkhuijsen et al. 2000). M31 is an early type spiral galaxy with a prominent bulge; at a 
distance of about $770 \mathrm{kpc}$ (Freedman \& Madore 1990; Stanek \& Garnavich 1998; van den Bergh 2000) - slightly increased compared to earlier estimates (de Vaucouleurs 1972). M31 is $30 \%$ to $50 \%$ larger than our own Galaxy (Hodge 1992) and extends over a few degrees on the sky. From rotation curves, the total mass within a $24 \mathrm{kpc}$ radius is estimated to $1.9 \times 10^{11} M_{\odot}$ (Rubin \& Ford 1970; Evans \& Wilkinson 2000 and references given there), with a mass/luminosity ratio of $12 \pm 1$. The total mass of M 31 could be as high as $1.2 \times 10^{12} M_{\odot}$ (Evans \& Wilkinson 2000), indicative of a significant dark halo. Based on the study of the rotation curves within a few arc-seconds from the center, the presence of a central black hole of $3 \times 10^{7} M_{\odot}$ is inferred (Kormendy \& Bender 1999 and references given there).

A prominent feature of M31 is the concentration of the interstellar medium in an annulus about $10 \mathrm{kpc}$ from the center, with additional spiral arm segments near $5 \mathrm{kpc}$ and extending out to $20 \mathrm{kpc}$. The ring structure is clearly evident in the optical, radio (e.g. Dame et al. 1993; Beck et al. 1998; Beck 2000) and infrared (Xu \& Helou 1996) domains, and is also indicated in the distribution of X-ray sources (Supper et al. 2001). Star formation in M 31 is largely concentrated in the annulus, where the cosmic ray rate is expected to be enhanced, also due to the exceptionally regular toroidal magnet field associated with the structure, with a strength around $5 \mu \mathrm{G}$ (Beck 1982; Berkhuijsen et al. 1993).

On the basis of CO (Dame et al. 1993; Berkhuijsen 1993) and $21 \mathrm{~cm}$ surveys (Cram et al. 1980), the total mass of interstellar gas is estimated to be similar for M 31 and the Galaxy; however, whereas in the Galaxy $\mathrm{H} 2$ and $\mathrm{HI}$ are found at roughly equal amounts, the total $\mathrm{H} 2$ mass of M 31 is estimated to about $3 \times 10^{8} M_{\odot}$, an order of magnitude below the mass in $\mathrm{HI}$ of about $4 \times 10^{9} M_{\odot}$. The star formation rate in M 31, in the range of 0.2 to $0.5 M_{\odot} \mathrm{yr}$ (Xu \& Helou 1996; Walterbos 1988), is significantly smaller than in the Milky Way (Mezger 1988). While optical searches for Supernova remnants in M 31 have resulted in a significant number of objects - well over 200 (Braun \& Walterbos 1993; Magnier et al. 1995) - the Supernova rate is also below that of our Galaxy. Braun \& Walterbos (1993) estimate a rate of 1.25/century for M 31, and Tamman et al. (1994) derive a rate of $1.2 /$ century, compared to about 2.5 /century for our Galaxy (Dragicevich et al. 1999 and references given there); Berkhuijsen (1984) finds similar ratios. However, the errors associated with these numbers are large, and equal rates in both Galaxies are not completely ruled out. The cosmic-ray electron flux in M31 has been estimated from the intensity of the radio continuum using equipartition arguments (e.g., Özel \& Berkhuijsen 1987), again resulting in a somewhat smaller density of relativistic electrons in M 31 .

M31 has been surveyed extensively in X-rays. First individual sources were resolved in the Einstein survey (van Speybroeck et al. 1979); including later reanalysis of the data, 108 sources were found. Two ROSAT surveys (Supper et al. 1997, 2001) resulted in 560 sources, with X-ray luminosities ranging from $4 \times 10^{35}$ to $4 \times 10^{38} \mathrm{erg} / \mathrm{s}$. The central region of M 31 was more recently surveyed by XMM (Shirey et al. 2001; Osborne et al. 2001) and 116 sources were located in the inner $30^{\prime}$. Chandra observation of the center region
(Garcia et al. 2000) resolved the nuclear source seen by Einstein and ROSAT in five discrete sources, one of which is most likely associated with the central black hole.

At gamma ray energies, an upper limit of $1.8 \times$ $10^{-8}$ photons $/ \mathrm{cm}^{2} \mathrm{~s}$ above $100 \mathrm{MeV}$ was derived from EGRET data (Blom et al. 1999); earlier EGRET results were given by Sreekumar et al. (1994). The limit implies that the gamma ray flux of M31 is less than 1/2 of that of the Milky Way, which is not too surprising if cosmic ray production is indeed linked to Supernova activity. Earlier upper limits on the TeV gamma-ray flux were given by Cawley et al. (1985) at a flux of $2.2 \times 10^{-11}$ photons $/ \mathrm{cm}^{2}$ above $400 \mathrm{GeV}$.

On basis of these characteristics, we will address the expectations concerning the visibility of $\mathrm{TeV}$ sources, in relation to the sensitivity of the instruments, which have reached the range of $10^{-12} / \mathrm{cm}^{2}$ s for the flux above $1 \mathrm{TeV}$, equivalent to a flux of about $5 \%$ of the flux detected from the Crab Nebula ${ }^{1}$. This flux sensitivity implies that only sources in M 31 with a TeV luminosity above a few $10^{38} \mathrm{erg} / \mathrm{s}$ have a chance to be detected. The visibility of gamma-ray sources in M 31 was so far mostly discussed in relation to the sub-GeV satellite energy range, see e.g. Özel \& Berkhuijsen (1986), Özel \& Fichtel (1988), Pavlidou \& Fields (2001).

Known Galactic $\mathrm{TeV}$ point sources are have peak fluxes at most in the range of the Crab flux. Scaling the flux according to the distance, such sources in M 31 generate a flux around $10^{-6}$ to $10^{-5}$ Crab units, well below the detection threshold of current instruments.

Another potential source is the diffuse radiation generated in interactions of cosmic rays with the interstellar medium. Optimistically assuming the same emissivity as in our own Galaxy, the predicted TeV flux is about $10^{-12} M_{6} / d_{\mathrm{kpc}}^{2} \mathrm{~cm}^{-2} \mathrm{~s}$, where $M_{6}$ is the target mass in units of $10^{6}$ solar masses, and $d_{\mathrm{kpc}}$ the distance in kpc (Aharonian 2001a). Expressed in Crab units, the expected flux is $6 \times 10^{-4}$, again below the sensitivity threshold. Scaling with the lower $S N$ rate in M 31 reduced predictions by another factor of a few (Pavlidou \& Fields 2001).

Like in AGN, the central black hole would be expected to generate high-energy gamma rays when accreting ambient matter. The limiting Eddington luminosity of the central black hole is $4 \times 10^{45} \mathrm{erg} / \mathrm{s}$, many orders of magnitude above the detection threshold. However, while the X-ray luminosity of the center of $\mathrm{M} 31$ - about $10^{39} \mathrm{erg} / \mathrm{s}$ in the $1-10 \mathrm{keV}$ range - is more or less in the range expected for normal galaxies, the radio emission is unusually low, indicating only normal or even sub-normal activity in the core of M31 (Yi \& Boughn 1999).

A final speculative source might be the annihilation radiation of neutralino dark matter accumulating near the center of M31 (see, e.g., Bergström et al. 1998, 1999, 2001; Ellis et al. 2002). In the framework of supersymmetric particles as the main constituent of non-baryonic dark matter in the universe, both monochromatic gamma-ray line emission and continuum emission is expected from the self-annihilation of the lightest

\footnotetext{
1 Here and in the following, we will use a flux of $1.75 \times$ $10^{-11} \mathrm{~cm}^{-2} \mathrm{~s}^{-1}$ above $1 \mathrm{TeV}$ for the Crab Nebula, taken from Aharonian et al. (2000).
} 
stable particle (LSP). Predicted flux levels are quite uncertain, and depend on how cuspy the distribution of dark matter near the center is - an issue still under much debate. Fluxes currently estimated from dark matter annihilation in our own Galactic center - and uncertain by up to two orders of magnitude are at or below the detection limits, and scale to fluxes below $10^{-4} \mathrm{Crab}$ units from the center of M 31 . The flux due to neutralino annihilation from M 31 in the CELESTE energy range $(>30 \mathrm{GeV}$ ) was recently discussed by Falvard et al. (2002), who argue in favor of a large dark matter component near the center of M 31, on the basis of rotation curves by Braun (1991). Still, the signal is at best marginally detectable. However, it has been speculated (Gondolo \& Silk 1999) that under the influence of a central black hole, as in our Galaxy or in M 31, the dark matter distribution should develop a spike, enhancing the annihilation rate by many orders of magnitude. On the other hand, it was argued by Merrit et al. (2002) that the spike is reduced due to mergers of dark matter clusters, and by Ullio et al. (2001) that a more detailed dynamical modeling shows only a much weakened spike.

In summary, one must conclude that such "conventional" $\mathrm{TeV}$ sources in M 31 are most likely not detectable with current instruments. On the other hand, as evidenced by the X-ray sources, there are objects in M31 in the relevant $10^{38} \mathrm{erg} / \mathrm{s} \mathrm{lu}$ minosity range; if the spectrum extends to highest energies, such sources would be visible in a survey, providing enough motivation for a measurement campaign with the HEGRA instrument.

\section{The HEGRA telescope system and the M 31 data set}

The HEGRA system of five imaging atmospheric Cherenkov telescopes is located on the Canarian Island of La Palma, at $2200 \mathrm{~m}$ asl. Each of the telescopes has a $8.5 \mathrm{~m}^{2}$ mirror and is equipped with a 271-pixel photomultiplier camera with a $4.3^{\circ}$ field of view. Cherenkov images in at least two telescopes are required to trigger the recording of event data; the energy threshold of the instrument is about $500 \mathrm{GeV}$ for sources in the zenith. From the stereoscopic Cherenkov images, the direction of the primary gamma-ray is reconstructed with an event-byevent precision of $0.1^{\circ}$. Cosmic ray background showers are strongly suppressed on the basis of image shapes. The effective detection area of the instrument is determined by the extent of the Cherenkov light pool and varies from $5 \times 10^{3} \mathrm{~m}^{2}$ at the nominal threshold to $10^{5} \mathrm{~m}^{2}$ well above threshold. The detection rate for gamma rays is rather uniform within the central $2^{\circ}$ of the field of view, and drops to $63 \%$ of its peak value at $1.8^{\circ}$ from the optical axis. Details about the telescope system and the analysis techniques are given in (Aharonian et al. 1999; Konopelko et al. 1999)

M 31 was observed in August, September and November 2001, using all five telescopes. In order to cover all of M31, observation time was distributed between three tracking positions, one centered on the core of M 31 at RA 0h 42' 44" DEC $41^{\circ} 16^{\prime} 9^{\prime} \cdot 12$, one displaced by $0.56^{\circ}$ to the SW at RA $0 \mathrm{~h} 40^{\prime} 30^{\prime \prime}$ DEC $40^{\circ} 39^{\prime} 0^{\prime \prime}$ and one displaced to the NE at RA $0 \mathrm{~h} 44^{\prime} 43^{\prime \prime}$ DEC $41^{\circ} 53^{\prime} 0^{\prime \prime}$. After data cleaning, $20.1 \mathrm{~h}$ of good observation time were selected, most of which were taken at Zenith angles below $25^{\circ}$. For calibration and reference, $9.7 \mathrm{~h}$ of Crab Nebula data taken in October and November were selected.

\section{Data analysis and search for $\mathrm{TeV}$ point sources}

The data analysis follows to a large extent the procedures developed for earlier surveys (Aharonian et al. 2001b, 2002), and concentrates on the search for sources which appear pointlike on the $0.1^{\circ}$ scale of the angular resolution of the instrument. The search for sources is carried out on a $6^{\circ}$ by $6^{\circ}$ grid with a spacing $0.031^{\circ}$ well below the angular resolution. For optimal rejection of cosmic-ray backgrounds, only events with at least four Cherenkov images (out of a maximum of five) were used, and gamma-ray candidates were selected by requiring on the mean scaled with $\bar{w}$ of images below 1.1; gamma rays generate a peak in $\bar{w}$ at 1.0 , with width 0.1 , whereas the distribution for the more diffuse cosmic ray images peaks at $\approx 1.6$ (see Fig. 1 of Aharonian et al. 2001b). For each grid point, gamma ray candidates with reconstructed directions within $0.143^{\circ}$, consistent within the angular resolution, were counted. Here, shower directions up to $1.8^{\circ}$ from the optical axis of the telescope system were accepted. Since no specific off-source data were taken, the expected background for each grid point is determined from the data itself, making use of the fact that the detection rate of the telescope system is (a) virtually flat near the center of the field of view, and (b) has an azimuthal symmetry with respect to the optical axis. Two methods for background estimation were applied: For search points more than $0.143^{\circ}$ from the optical axis, three regions the same size as the signal region, but rotated by $90^{\circ}, 180^{\circ}$ and $270^{\circ}$ in azimuth with respect to the optical axis were used to estimate the background. This procedure cannot be used for search regions near the optical axis, since signal region and background regions overlap. Instead, a circular background region was chosen, centered on the signal region, with an inner radius of twice the radius of the signal region, and an outer radius adjusted such that the area of the background region is three times the area of the signal region. A cross-check of both methods in the regions of overlap indicates only negligible systematic differences between both methods. In the following we therefore give results for the ring model. Figure 1a shows the distribution of detected gamma-ray candidates from $M 31$, selected by a $\bar{w}<1.1$ cut. Figure $1 \mathrm{~b}$ gives the predicted number of background events. No obvious excess of signal events is visible at any grid point. The significance for each grid point is then calculated based on Li \& Ma (1983), and is shown in Fig. 1c. The distribution of significances for all grid point approximates a Gaussian distribution with unit width, as expected in the absence of a genuine signal (Fig. 2); the point with the highest significance of $3.5 \sigma$ is well compatible with the expected tail of the Gaussian distribution, and should be interpreted as an upward fluctuation.

We conclude that there is no statistically significant indication of a $\mathrm{TeV}$ gamma ray point source in $\mathrm{M} 31$, and proceed to extract upper limits for the source flux. To derive the limits, first the $99 \%$ confidence level on the number of excess events is calculated according to Helene (1983). The number is then 


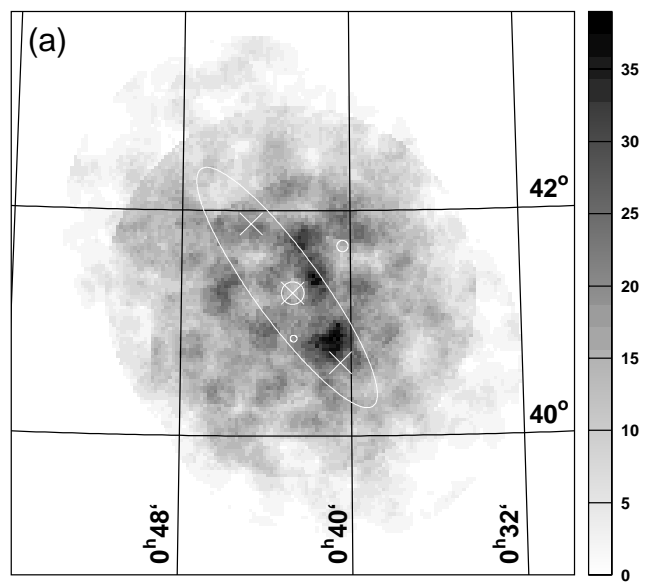

Number of $\mathrm{ON}$ events

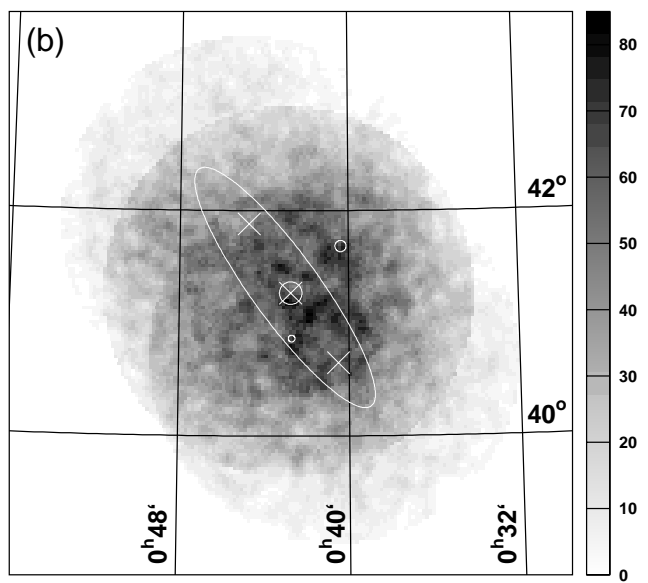

Number of OFF events

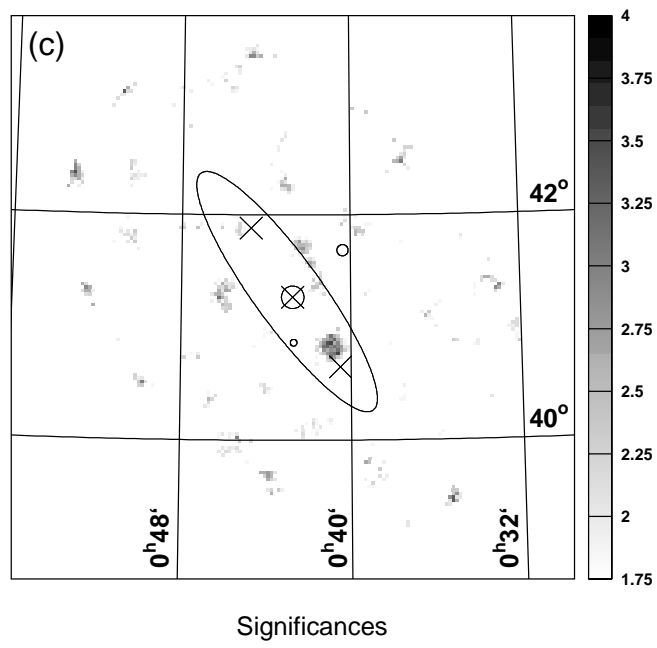

Fig. 1. (a) Number of gamma ray candidates for each point of the search grid, (b) estimated number of background events, (c) significance for an excess of signal events. For reference, the three tracking locations are indicated by crosses, the locations of the center of M 31 and of the $10 \mathrm{kpc}$ dust ring are indicated. The small circles indicate the positions of M 32 and NGC 205.

corrected for the $28 \%$ acceptance loss of the angular cut of $0.143^{\circ}$ relative to the direction of the source. The number of

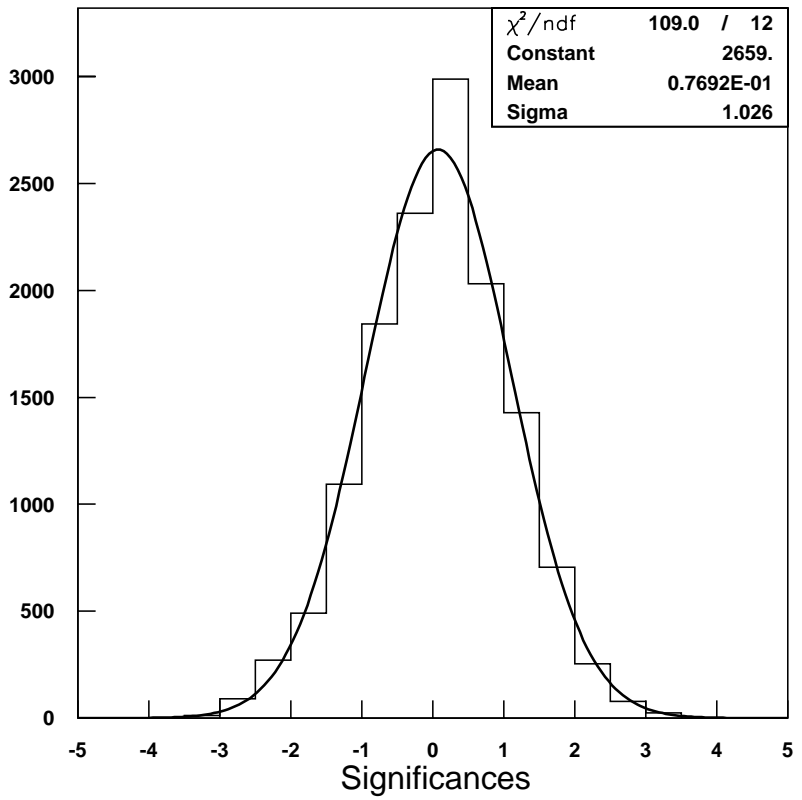

Fig. 2. Distribution of the significance for an excess at the points of the search grid. We note that the significances derived for adjacent points are correlated, since the spacing of points is of the same scale as the experimental resolution. There is no point above $3.5 \sigma$ and the distribution is well represented by a Gaussian of mean 0.08 and width 1.026 .

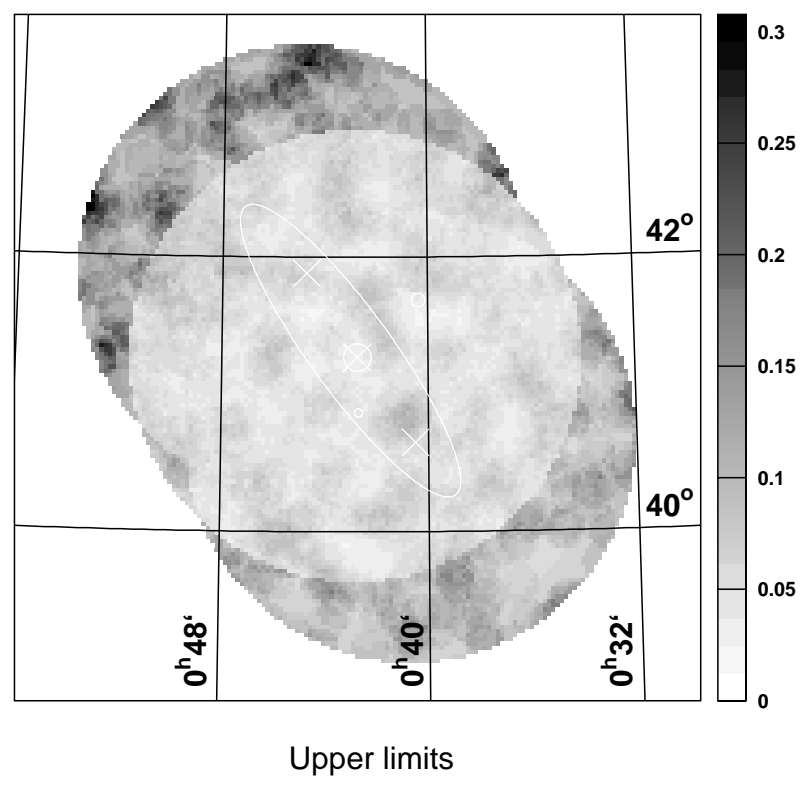

Fig. 3. Upper limits on the flux from $\mathrm{TeV}$ point sources in M31, expressed in units of the Crab flux. For reference, the locations of the center of M31, the $10 \mathrm{kpc}$ dust ring and the two companion galaxies M 32 and NGC 205 are indicated. Tracking positions are indicated by crosses.

upper limit counts is normalized to the expected rate detected from the Crab nebula at the relevant zenith angle; since the Crab observations were carried out with the source positioned at a fixed angle of $0.5^{\circ}$ from the optical axis, the dependence of detection rate on the position within the field of view was 
corrected based on Monte Carlo simulations (see Fig. 3 of Aharonian et al. 2001b). The limits are given in units of the Crab flux and refer to a characteristic energy of $1 \mathrm{TeV}$. The limits are calculated assuming the Crab energy spectrum, but are relatively insensitive to this assumption; a variation in spectral index of \pm 0.5 changes the flux limits by less than $10 \%$.

The resulting flux limits are shown in Fig. 3, and range from $3.3 \%$ of the Crab flux for the center of M 31 to about $30 \%$ at the periphery. For two other objects in the survey range, M 32 and NGC 205, limits of $4.4 \%$ and $2.8 \%$ of the Crab flux are derived, respectively.

\section{Search for supersymmetric dark matter in M 31}

Beyond the general survey, the good energy resolution of the HEGRA telescope system allows a dedicated search for supersymmetric dark matter in M31, looking for the line emission from the self-annihilation of the lightest stable particle (LSP). We have searched for line emission in the energy spectrum from the central part of M 31 ( $r<1.4 \mathrm{kpc}$ ), and compare the results with predictions of the flux of gamma-rays from the annihilation of neutralinos $\left(\chi_{0}\right)$ in the framework of the minimal supersymmetric standard model (MSSM) using a spatial distribution of the radial density of neutralinos in M31 in concordance with the measured rotation curve (Braun 1991; Falvard et al. 2002).

The event selection and background estimate for the spectral analysis deviates from the approach taken for the survey for point-sources in M31. Events with three or more Cherenkov images were used. In order to reduce the background from isotropic cosmic ray events, a tight cut on the direction has been chosen for the central part of M 31. Only events from within a cone of radius $0.105^{\circ}$ are accepted. At a distance of $770 \mathrm{kpc}$ this corresponds to the inner $1.4 \mathrm{kpc}$ of M31. The cut on $\bar{w}$ remains the same $(\bar{w}<1.1)$. The sensitivity for line emission depends on the energy resolution of the detector. For this analysis, an improved energy reconstruction algorithm as described in Hofmann et al. (2000) has been applied. The relative energy resolution $(\Delta E / E)$ reaches $10 \%$ for a wide energy band from the threshold of $500 \mathrm{GeV}$ up to $10 \mathrm{TeV}$. The search bin in energy is $12 \%$ wide, concentrating on the central section of a monoenergetic line in order to achieve best signal to noise ratios. We have not attempted to optimize the search bins individually for the different energy regions; the optimal choice would have to take the diminishing event statistics at higher energies into account. The energy bins are equally spaced on a logarithmic scale such that the bin centers are separated by 0.025 on a decadic logarithmic scale making the bins correlated.

The background expectation of the measurement is determined using seven independent OFF-regions with similar acceptance to the ON-region. For the calculation of upper limits on the number of excess events $N_{\gamma}^{99 \%}$, the $99 \%$ c.l. upper limits were calucated according to Helene (1983), and an upper limit on the rate from a $\gamma$-ray line was derived. The corresponding flux limits were calculated using collection areas $A_{\text {eff }}(E, \theta)$ derived from Monte Carlo simulations (Konopelko et al. 1999) applying the same reconstruction methods and event selection

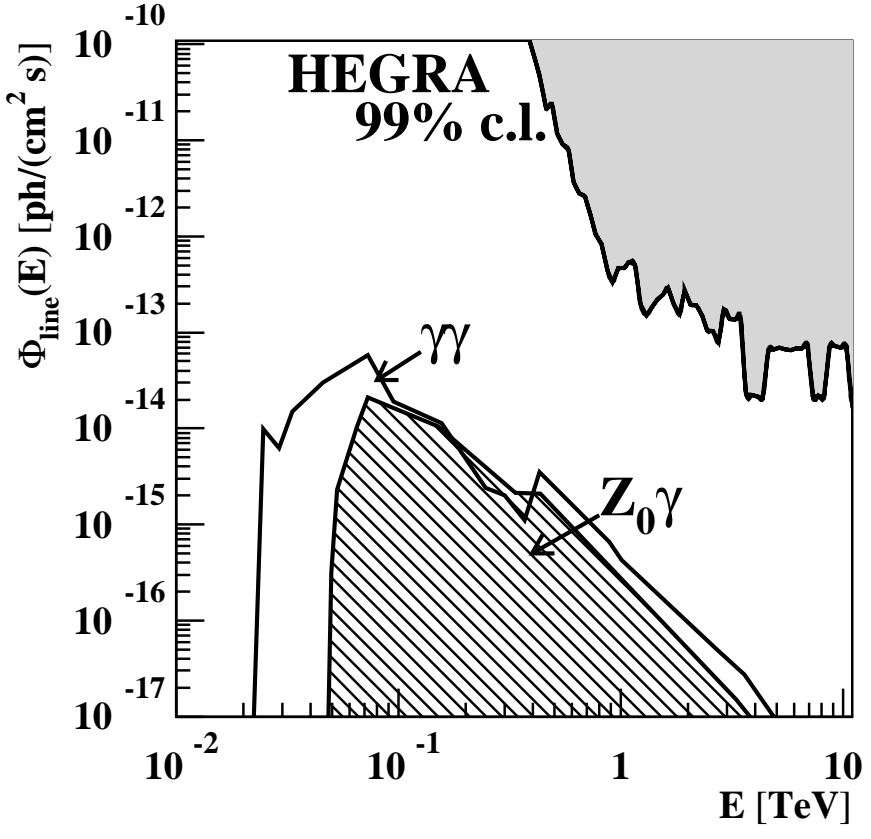

Fig. 4. Exclusion region (grey shaded region) for the the gamma-ray line flux from the center of M31. The two sets of curves labeled $\gamma \gamma$ and $Z^{0} \gamma$ indicate the upper range of model predictions for the minimal supersymmetric models calculated in Bergström et al. (1998). The dark matter halo model for M 31 has been taken from Falvard et al. (2002, Table 1), using a mass-to-light ratio of 3.5 for the bulge and 2.5 for the disk.

as for the data analysis. The resulting exclusion region is indicated in Fig. 4 as the grey shaded region.

A prediction for the line flux emission was derived using scans of the 7-parameter space of MSSM performed by Bergström et al. (1998). The expected flux is given by Eq. (6) of Falvard et al. (2002) or the equivalent Eq. (13) of Bergström et al. (1998) (scaled to the distance to M31):

$$
\begin{aligned}
I_{\gamma}= & \left(3.18 \times 10^{-13} \text { photons } \mathrm{cm}^{-2} \mathrm{~s}^{-1}\right)\left(\frac{\langle\sigma v\rangle N_{\gamma}}{10^{-25} \mathrm{~cm}^{3} \mathrm{~s}^{-1}}\right) \\
& \times\left(\frac{500 \mathrm{GeV}}{m_{\chi}}\right)^{2} \Sigma_{19} .
\end{aligned}
$$

Apart from trivial factors, it depends on the velocity-weighted annihilation cross section $\langle\sigma v\rangle$ and on the line-of-sight and solid-angle integrated squared LSP mass density $\rho$

$\Sigma=\iint \rho^{2} \mathrm{~d} s \mathrm{~d} \Omega$

written as $\Sigma_{19}$ in units of $10^{19} \mathrm{GeV}^{2} \mathrm{~cm}^{-5}$. The envelope of the allowed $\langle\sigma v\rangle$ vs. $M_{\chi}$ space was used as given in Figs. 1 and 2 of Bergström et al. (1998). The unitless parameter $\Sigma_{19} \approx 1.7$ corresponds to $\Sigma_{19}=3$ of Falvard et al. (2002), scaled to the inner $1.4 \mathrm{kpc}$ on the basis of the $\rho(r)$ distributions of Fig. $3 \mathrm{~b}$ of Falvard et al. (2002) and using a distance of $770 \mathrm{kpc}$. This value represents the upper range of the different models for the dark matter halo of M31 discussed in this reference. The predicted line flux is given in Fig. 4. We have indicated the 
two individual final states with line emission $\left(\chi^{0} \chi^{0} \rightarrow \gamma \gamma\right.$ and $\left.\chi^{0} \chi^{0} \rightarrow Z^{0} \gamma\right)$ in the figure. The model prediction given here is based upon smoothly distributed dark matter. Within this model, no signal from M31 with the current sensitivity of Cherenkov detectors is to be expected. Very favorable conditions as for example clumpiness of dark matter would lead to a considerable increase of the annihilation rate (Bergström et al. 1999; Taylor \& Silk 2002). It is conceivable that the flux level of some models would be become detectable by tuning the distribution of the dark matter halo and by invoking mechanisms to allow for larger cross sections e.g. quintessence (Salati 2002) or anomaly-mediated supersymmetry breaking scenarios (Ullio 2001). However, in the absence of a signal, no exotic speculations are required.

\section{Concluding remarks}

For the first time, the Andromeda galaxy was surveyed for point-like sources of $\mathrm{TeV}$ gamma rays. No sources were detected at levels exceeding a few percent of the Crab flux, equivalent to a source strength of about $3 \times 10^{38} \mathrm{erg} / \mathrm{s}$ above $1 \mathrm{TeV}$. The fact that limits within of a few percent of the Crab flux were achieved with only $20 \mathrm{~h}$ of observation time distributed over three locations indicates the enormous progress of the detection technique during the last years. While TeV sources of a detectable strength are well within the range of source energetics, the lack of such sources does not come as a big surprise, given the discussion presented in the introduction and the comparison with Galactic TeV sources. The observations rule out a new and hitherto undiscovered population of very strong VHE sources in normal galaxies.

We have specifically searched for the first time for a gamma-ray line from M31 with an energy resolution of $\Delta E / E=10 \%$. We have not found any indication for a signal, setting upper limits at the level of $10^{-13} \mathrm{~cm}^{-2} \mathrm{~s}^{-1}$ above $1 \mathrm{TeV}$. The expectation for the $\gamma$-ray line flux from M 31 is by 2 orders of magnitude lower than the sensitivity reached with the current instruments. However, with a lower energy threshold $(100 \mathrm{GeV})$ and better sensitivity combined with a similar energy resolution as achieved by next-generation experiments like H.E.S.S. and VERITAS it will be possible to probe the MSSM parameter space for the Galactic center and nearby galaxies.

Acknowledgements. The support of the HEGRA experiment by the German Ministry for Education and Research BMBF and by the Spanish Research Council CICYT is acknowledged. We are grateful to the Instituto de Astrofísica de Canarias for the use of the site and for providing excellent working conditions. We gratefully acknowledge the technical support staff of Heidelberg, Kiel, and Munich.

\section{References}

Aharonian, F. A., Akhperjanian, A. G., Barrio, J. A., et al. 1999, A\&A, 349,11

Aharonian, F. A., Akhperjanian, A. G., Barrio, J. A., et al. 2000, ApJ, 539, 317

Aharonian, F. A. 2001a, Space Sci. Rev., 99, 187
Aharonian, F. A., Akhperjanian, A. G., Barrio, J. A., et al. 2001b, A\&A, 375, 1008

Aharonian, F. A., Akhperjanian, A. G., Barrio, J. A., et al. 2002, submitted [astro-ph/0209360]

Beck, R. 1982, A\&A, 106, 121

Beck, R., Berkhuijsen, E. M., \& Hoernes, P. 1998, A\&AS, 129, 329

Beck, R. 2000, in The Interstellar Medium in M 31 and M33, 232, WE-Haraeus Seminar, ed. E. M. Berkhuijsen, R. Beck, \& R. A. M. Walterbos (Aachen: Shaker), 171

Bergström, L., Edsjö, J., \& Gunnarson, C. 2001, Phys. Rev. D, 63, 083515

Bergström, L., Ullio, P., \& Buckley, J. H. 1998, Astropart. Phys., 9, 137

Bergström, L, Edsjö, J., Gondolo, P., \& Ullio, P. 1999, Phys. Rev. D, 59, 043506

Berkhuijsen, E. M. 1984, A\&A, 140, 431

Berkhuijsen, E. M., Bajaja, E., \& Beck, R. 1993, A\&A, 279, 359

Berkhuijsen, E. M., Beck, R., \& Walterbos, R. A. M. (ed.) 2000, The Interstellar Medium in M 31 and M33, 232, WE-Haraeus Seminar (Aachen: Shaker)

Blom, J. J., Paglione, T. A. D., \& Carraminana, A. 1999, ApJ, 516, 744

Braun, R. 1991, ApJ, 372, 54

Braun, R., \& Walterboos, R. A. M. 1993, A\&AS, 98, 327

Cawley, M. F., Fegan, D. J., Gibbs, K., et al. 1985, Proc. 19th Int. Cosmic Ray Conf., 1, 264

Cram, T. R., Roberts, M. S., \& Whitehurst, R. N. 1980, A\&AS, 40, 215

Dame, T. M., Koper, E., Israel, F. P., \& Thaddeus, P. 1993, ApJ, 418, 730

Daum, A., Hermann, G., Heß, N., Hofmann, W., et al. 1997, Astropart. Phys., 8,1

de Vaucouleurs, G., \& de Vaucouleurs, A. 1972, Mem. Roy. Astron. Soc., 77, 40

Dragicevich, P. M., Blair, D. G., \& Burman, R. R. 1999, MNRAS, 302, 693

Ellis, J. R., Feng, J. L., Ferstl, A., et al. 2002, Eur. Phys. J. C, 24, 311

Evans, N. W., \& Wilkinson, M. I. 2000, MNRAS, 316, 929

Falvard, A., Giraud, E., Jacholkowska, A., et al. 2002 [astro-ph/0210184]

Freedman, W. L., \& Madore, B. F. 1990, ApJ, 365, 186

Garcia, M. R., Murray, S. S., Primini, F. A., et al. 2000, ApJ, 537, L23

Gondolo, P., \& Silk, J. 1999, PRL, 83, 1719

Helene, O. 1983, Nucl. Instr. Meth., 212, 319

Hodge, P. 1992, The Andromeda Galaxy (Dordrecht: Kluwer)

Hofmann, W., Lampeitl, H., Konopelko, A., \& Krawczynski, H. 2000, Astropart. Phys., 12, 207

Konopelko, A., Hemberger, M., Aharonian, F., Daum, A., et al. 1999, Astropart. Phys., 10, 275

Kormendy, J., \& Bender, R. 1999, ApJ, 522, 772

Li, T., \& Ma, Y. 1983, ApJ, 272, 317

Magnier, E. A., Prins, S., van Paradijs, J., et al. 1995, A\&AS, 114, 215

Merrit, D., Milosavljevic, M., Verde, L., \& Jimenez, R. 2002, PRL, 88,191301

Mezger, P. G. 1988, in Galactic and Extragalactic Star Formation, ed. R. E. Pudritz, \& M. Fich (Dordrecht: Kluwer), 227

Özel, M. E., \& Berkhuijsen, E. M. 1987, A\&A, 172, 378

Özel, M. E., \& Fichtel, C. E. 1988, ApJ, 335, 135

Osborne, J. P., Borozdin, K. N., Trudolyubov, S. P., et al. 2001, A\&A, 378,800

Pavlidou, V., \& Fields, B. D. 2001, ApJ, 558, 63

Rubin, V. C., \& Ford, W. K. 1970, ApJ, 159, 379

Salati, P. 2002 [astro-ph/0207396] 
Shirey, R., Soria, R., Borozdin, K., et al. 2001, A\&A, 365, L195 Sreekumar, P., Bertsch, D. L., Dingus, B. L., et al. 1994, ApJ, 426, 105 Stanek, K. Z., \& Garnavich, P. M. 1998, ApJ, 503, L131

Supper, R., Hasinger, G., Pietsch, W., et al. 1997, A\&A, 317, 328

Supper, R., Hasinger, G., Lewin, W. H. G., et al. 2001, A\&A, 373, 63 Tammann, G. A., Löffler, W., \& Schröder, A. 1994, ApJS, 92, 487

Taylor, J. E., \& Silk, J. 2002, MNRAS, submitted [astro-ph/0207299]

Ullio, P. 2001, JHEP, 0106, 053

Ullio, P., Zhoa, H., \& Kamionkowski, M. 2001, Phys. Rev. D, 64, Yi, I., \& Boughn, S. P. 1999, ApJ, 515, 576

van den Bergh, S. 2000, The Galaxies of the Local Group (Cambridge Univ. Press)

Van Speybroeck, L., Epstein, A., Forman, W., et al. 1979, ApJ, 234, L45

Weekes, T. C. 2001, High Energy Gamma-Ray Astronomy, AIP Conf. Proc. 558, 15

Walterbos, R. A. M. 1988, in Galactic and Extragalactic Star Formation, ed. R. E. Pudritz, \& M. Fich (Dordrecht: Kluwer), 361

Xu, C., \& Helou, G. 1996, ApJ, 456, 152 043504 